\title{
Endoscopic Approach of the Inverted Papilloma - a continuos challenge for the ENT practitioner
}

\author{
Vlad Andrei Budu' ${ }^{1 *}$, Ioan Alexandru Bulescu ${ }^{2}$, Mihail Tusaliu ${ }^{2}$ \\ ${ }^{1}$ University of Medicine and Pharmacy, Romania \\ ${ }^{2}$ University of Medicine and Pharmacy Carol Davila Bucharest, Romania
}

Submission: February 04, 2017; Published: February 07, 2017

*Corresponding author: Vlad Andrei Budu, University of Medicine and Pharmacy, Carol Davila, 14 Eroilor Blvd., Bucharest, Romania, Tel: +40788413218; Email: vladbudu@yahoo.com

\section{Editorial}

\section{Introduction}

Inverted papilloma is a benign tumor arising from the epithelium of the nose and sinuses with a slow growing pattern and a confirmed viral etiology (Human Papilloma Virus) in more than $80 \%$ of patients. The main characteristics of inverted papilloma are represented by important distruction of surrounding structures, high risk of reccurence and malignant degeneration. In more than 180 cases of inverted papilloma which I approached endoscopically, I got the experience to emphasize on some tips and tricks regarding the diagnosis and surgical treatment of this sinonasal tumor.

Tips and tricks in inverted papilloma Unilateral lesion: Phisical and endoscopic exam of the nasal cavity reveals a raddish gray polipoid mass with friable apparence and bleeding history which usually causes unilateral nasal obstruction and epistaxis. CT-scan is considered to be the gold-standard investigation and provides complete data on the dimensions and extensions of the tumor, the involvment of the bony structures and the bony stalk or focal hyperostosis which correspond to the origin of the tumor (Figures 1 \& 2).

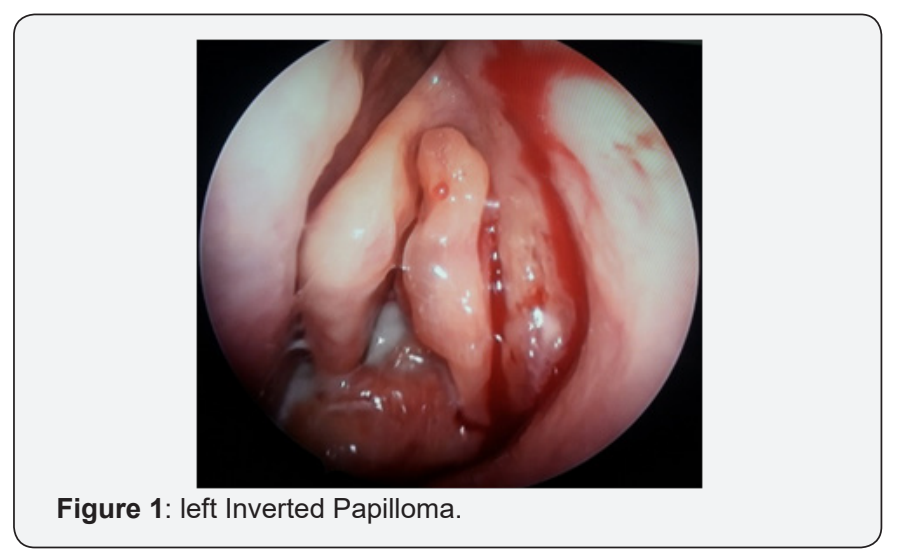

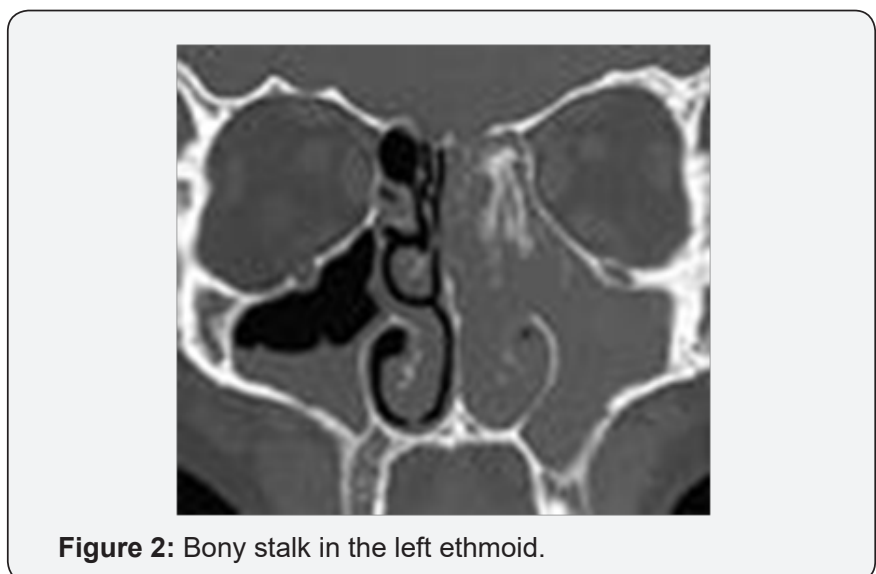

Staging: In all our patients we used Krouse Staging System for inverted papilloma and that allowed us to decide the proper surgical technique for each case in particular. In our series the most frecquent situation was Krouse Stage III, and we could perform an accurate surgical procedure for complete removal of the tumor.

Endoscopic Sinus Surgery: Usually, while dealing with an inverted papilloma (stage I-III) we do not perform routinely a biopsy in before surgery, our surgical protocol is to harvest tumoral fragments for frozen sections. Endoscopic sinus surgery assures complete removal of the tumor and the adjacent mucosa, drilling of the underlying bone and mandatory the bony stalk (origin of the inverted papilloma) (Figures $3 \& 4$ ). In Krouse Stages II and III medial maxillectomy is the gold standard for surgical approach, completed when necessary with ethmoidectomy (anterior or total), frontal recess and frontal sinus endoscopic approach and sphenoidotomy. It is not indicated an endoscopic approach for inverted papilloma situated in the lateral part of the frontal sinus. 


\section{Global Journal of Otolaryngology}
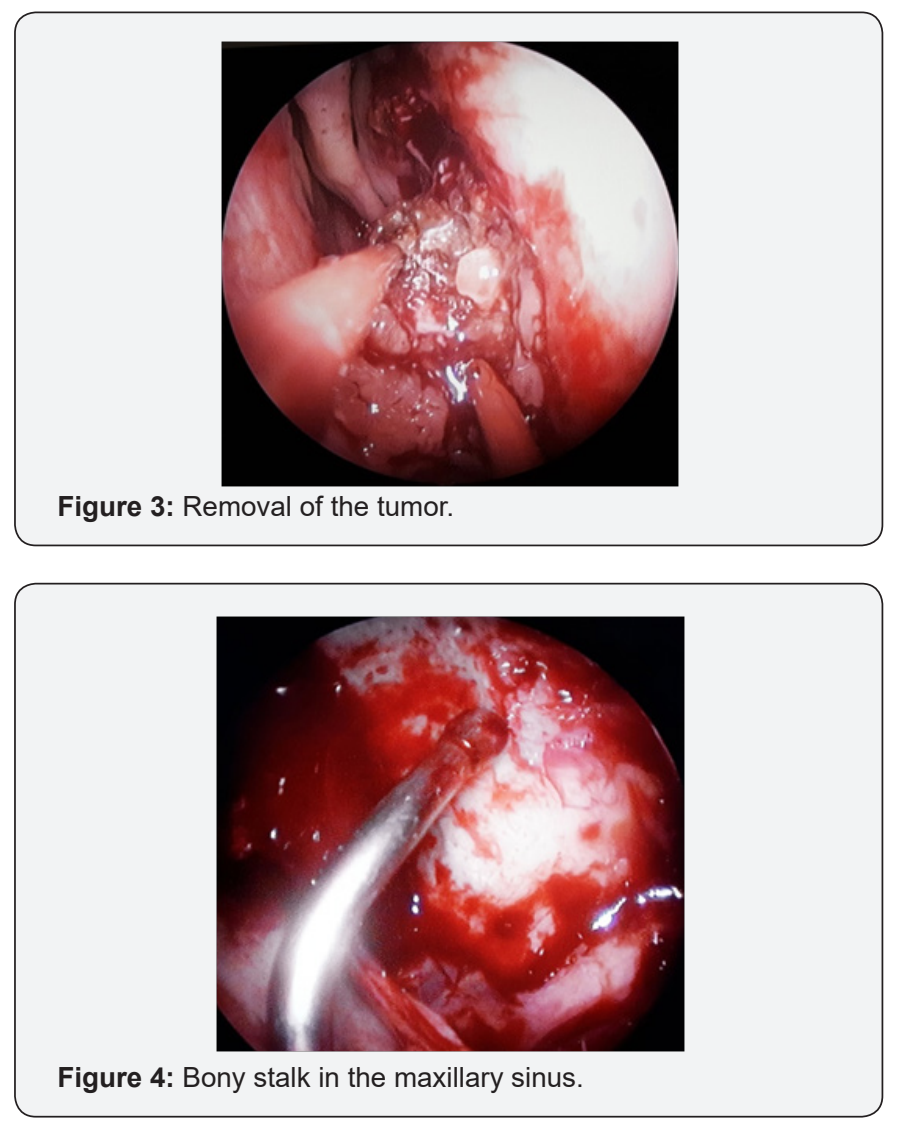

Human Papilloma Virus: We performed a HPV-genotype from every removed specimen in order to find the presence of viral DNA at the tumor site. HPV is a virus with epithelial tropism which determins premalignant or malignant lesions in anogenital tract, but in nasosinusal region it is associated to a high risk of reccurence and malignant transformation of the inverted papilloma.

\section{Reccurence}

The most frecquent question regarding inverted papilloma is if we are dealing with reccurences of left overs. We had in our series reapparence of the tumor after precise surgical tehnique and also in early stages. There was no reason for reccurence in that particular cases so we are thinking more often as microscopic fragments of tumor or mucosa with parts of viralDNA, which can not be seen during surgery, but exist and react just like tumoral left-over. We also noticed in our series that patients which already had a reccurence, have a greater risk for another one. So reccurence can be explained by the presence of intracellular viral-DNA, as a constant HPV infection in the sinonasal mucosa.

\section{Malignant degeneration}

It was thought in the past that malignant transformation of the inverted papilloma is due to the numerous surgical procedures suffered by the patient. Nowadays we find and confirm in lab (in situ DNA hybridization) in tumor fragments or surrounding mucosa low risk subtypes (HPV6, HPV11) and high risk subtypes (HPV16, HPV18) which are responsable for malignant transformation of the inverted papilloma.

In this challenge with the inverted papilloma I faced therapeutical limits:

a) treatment begins when the tumor is on site and becomes simptomatologic

b) in most of the cases surgical treatment is not enough so we developed a protocol which consists in a multimodal treatment: surgery, antiviral treatment (Interferon, Cidofovir,etc), and antiangiogenetic factors (Bevacizumab)

c) we do not have yet no protocol to prevent malignant degeneration of the inverted papilloma

Instead of conclusion I will launch some questions:

1) how can we manage HPV infection prophilaxis in sinonasal pathology?

2) how can we predict which of our patient with HPV infection in nasal mucosa will develop inverted papilloma?

3) which is the best therapeutic solution for a patient with complete removal of an inverted papilloma with high risk HPV genotype?
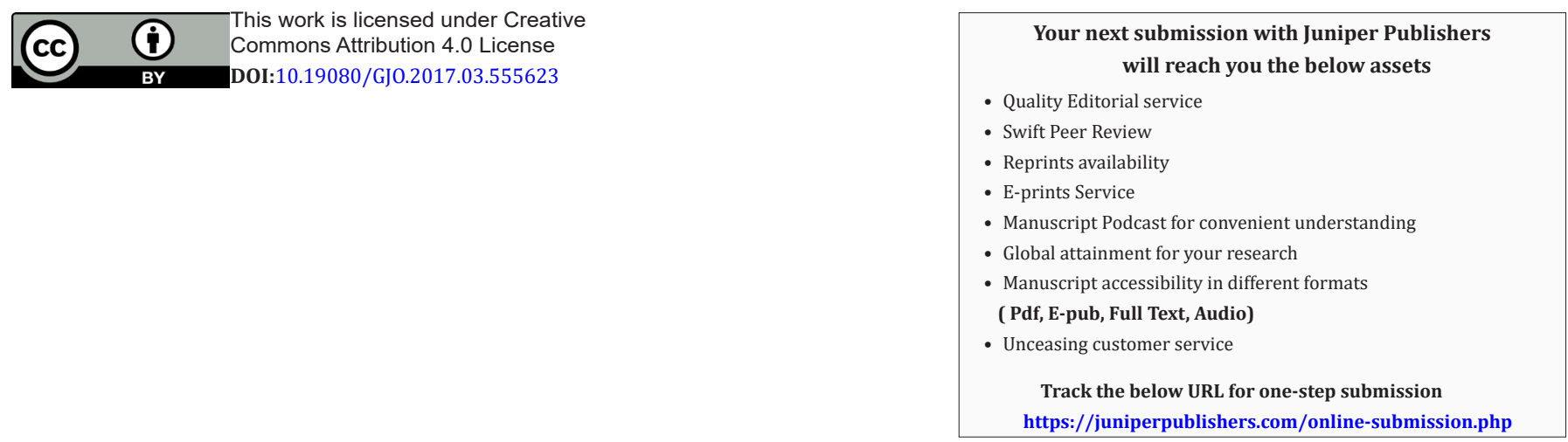